\title{
DIRAS2 is Associated with Adult ADHD, Related Traits, and Co-Morbid Disorders
}

\begin{abstract}
Andreas Reif*, , T Trang Nguyen ${ }^{2,24}$, Lena Weißflog,24, Christian P Jacob ${ }^{1,3}$, Marcel Romanos ${ }^{4}$, Tobias J Renner ${ }^{4}$, Henriette N Buttenschon ${ }^{5}$, Sarah Kittel-Schneider', Alexandra Gessner', Heike Weber', Maria Neuner', Silke Gross-Lesch ${ }^{3}$, Karin Zamzow ${ }^{2}$, Susanne Kreiker ${ }^{3,4}$, Susanne Walitza ${ }^{6}$, Jobst Meyer ${ }^{7}$, Christine M Freitag $^{8}$, Rosa Bosch", Miquel Casas', Nuria Gómez", Marta Ribasès', Mónica Bayès ${ }^{10}$, Jan K Buitelaar ' ', Lambertus ALM Kiemeney ${ }^{12}$, JJ Sandra Kooij ${ }^{13}$, Cees C Kan ${ }^{14}$, Martine Hoogman ${ }^{14}$, Stefan Johansson ${ }^{15,16}$, Kaya K Jacobsen ${ }^{15,16}$, Per M Knappskog ${ }^{15}$, Ole B Fasmer ${ }^{17}$, Phil Asherson ${ }^{18}$, Andreas Warnke ${ }^{4}$, Hans-Jörgen Grabe ${ }^{19}$, Jessie Mahler ${ }^{19}$, Alexander Teumer ${ }^{20}$, Henry Völzke ${ }^{21}$, Ole N Mors ${ }^{5}$, Helmut Schäfer ${ }^{2}$, Josep Antoni Ramos-Quiroga9', Bru Cormand ${ }^{22}$, Jan Haavik ${ }^{16,17}$, Barbara Franke ${ }^{14,23}$ and Klaus-Peter Lesch ${ }^{3}$

'Department of Psychiatry, Psychiatric Neurobiology and Bipolar Disorder Program, Psychosomatics and Psychotherapy, University of Würzburg, Würzburg, Germany; ${ }^{2}$ Institute of Medical Biometry and Epidemiology, University of Marburg, Marburg, Germany; ${ }^{3}$ Department of Psychiatry, ADHD Clinical Research Network, Molecular Psychiatry Laboratory of Translational Neuroscience; Psychosomatics and Psychotherapy, University of Wuerzburg, Wuerzburg, Germany; ${ }^{4}$ Department of Child and Adolescent Psychiatry, Psychosomatics and Psychotherapy, University of Würzburg, Würzburg, Germany; ${ }^{5}$ Centre for Psychiatric Research, Aarhus University Hospital, Risskov, Denmark; ${ }^{6}$ Department of Child and Adolescent Psychiatry, University of Zuerich, Zuerich, Switzerland; ' Department of Neurobehavioral Genetics, University of Trier, Institute of Psychobiology, Trier, Germany; ${ }^{8}$ Department of Child and Adolescent Psychiatry, Goethe University Frankfurt am Main, Frankfurt, Germany; ${ }^{9}$ Department of Psychiatry, Hospital Universitari Vall d'Hebron, Barcelona, Catalonia, Spain; ${ }^{10}$ Centro Nacional de Análisis Genómico (CNAG), Barcelona, Catalonia, Spain; " Department of Cognitive Neuroscience, Donders Institute for Brain, Cognition and Behavior, Radboud University Nijmegen Medical Centre, Nijmegen, The Netherlands; ${ }^{12}$ Department of Epidemiology, Biostatistics and HTA, Radboud University Nijmegen Medical Centre, Nijmegen, The Netherlands; ${ }^{3}$ PsyQ, Psycho-Medical Programs, Program Adult ADHD, The Hague, The Netherlands; ${ }^{14}$ Department of Psychiatry, Donders Institute for Brain, Cognition and Behavior, Radboud University Nijmegen Medical Centre, Nijmegen, The Netherlands; ${ }^{15}$ Department of Medical Genetics and Molecular Medicine, Haukeland University Hospital, Bergen, Norway; ${ }^{16}$ Department of Biomedicine, University of Bergen, Bergen, Norway; ${ }^{17}$ Division of Psychiatry, Haukeland University Hospital, Bergen, Norway; ${ }^{18}$ MRC Social Genetic Developmental and Psychiatry Centre, Institute of Psychiatry, London, UK; ${ }^{19}$ Department of Psychiatry and Psychotherapy, University of Greifswald, Greifswald, Germany; ${ }^{20}$ Interfaculty Institute for Genetics and Functional Genomics, University of Greifswald, Greifswald, Germany; ${ }^{21}$ Institute for Community Medicine, University of Greifswald, Greifswald, Germany; ${ }^{22}$ Departament de Genètica, Facultat de Biologia, Universitat de Barcelona, and CIBER Enfermedades Raras, and Institut de Biomedicina de la Universitat de Barcelona (IBUB), Catalonia, Spain;

${ }^{23}$ Department of Human Genetics, Radboud University Nijmegen Medical Center, Nijmegen, The Netherlands
\end{abstract}

Several linkage analyses implicated the chromosome $9 q 22$ region in attention deficithyperactivity disorder (ADHD), a neurodevelopmental disease with remarkable persistence into adulthood. This locus contains the brain-expressed GTP-binding RAS-like 2 gene (DIRAS2) thought to regulate neurogenesis. As DIRAS2 is a positional and functional ADHD candidate gene, we conducted an association study in 600 patients suffering from adult ADHD (aADHD) and 420 controls. Replication samples consisted of I035 aADHD patients and I 38 I controls, as well as I 66 families with a child affected from childhood ADHD. Given the high degree of co-morbidity with ADHD, we also investigated patients suffering from bipolar disorder (BD) ( $n=336)$ or personality disorders (PDs) $(n=622)$. Twelve single-nucleotide polymorphisms (SNPs) covering the structural gene and the transcriptional control region of DIRAS2 were analyzed. Four SNPs and two haplotype blocks showed evidence of association with $\mathrm{ADHD}$, with nominal $p$-values ranging from $p=0.006$ to $p=0.05$. In the adult replication samples, we obtained a consistent effect of $r$ I $4 \mid 2005$ and of a risk haplotype containing the promoter region $(p=0.026)$. Meta-analysis resulted in a significant common OR of $1.12(p=0.04)$ for rs /4I2005 and confirmed association with the promoter risk haplotype $(O R=1.45, p=0.0003)$. Subsequent analysis in nuclear families with childhood ADHD again showed an association of the promoter haplotype block $(p=0.02)$. rs $|4| 2005$ also increased risk toward BD $(p=0.026)$ and cluster B PD $(p=0.031)$. Additional SNPs showed association with personality scores $(p=0.008-0.048)$. Converging lines of evidence implicate genetic variance in the promoter region of DIRAS2 in the etiology of ADHD and co-morbid impulsive disorders. Neuropsychophamacology (20 I I) 36, 2318-2327; doi: 10.1038/npp.20I I.120; published online I 3 July 20 I I

Keywords: adult ADHD; linkage; genome-wide association; ras pathway; association study; bipolar disorder

*Correspondence: Professor A Reif, Department of Psychiatry, Psychiatric Neurobiology and Bipolar Disorder Program, Department of Psychiatry, Psychosomatics and Psychotherapy, University of Würzburg, Füchsleinstr. I5, D-97080 Würzburg, Germany, Tel: + 49 93I 20I 76402;

Fax: + 49 931 201 76403, E-mail: reif_a@klinik.uni-wuerzburg.de

${ }^{24}$ These authors contributed equally to this work.

Received 27 November 20 I0; revised II April 20 I I; accepted 24 May 20 I I 


\section{INTRODUCTION}

Attention deficit/hyperactivity disorder (ADHD) is a prevalent psychiatric condition in childhood associated with substantial impairment. In up to $30 \%$ of all affected cases, the disorder persists into adulthood (adult ADHD, aADHD; Faraone et al, 2000), although the core symptoms become less prominent, as adaptive mechanisms interfere with the presentation of the disease and as co-morbid conditions modify or even dominate the clinical picture. The latter include substance use disorders, affective, and personality disorders (PDs) (Jacob et al, 2007). Furthermore, there is accumulating evidence for neurobiological overlap with bipolar disorder (BD), with a high percentage of aADHD patients also suffering from $\mathrm{BD}$ (Klassen et al, 2010; Wingo and Ghaemi, 2007) and even more from bipolar symptoms on the dimensional level (Halmoy et al, 2010). Conversely, approximately $10 \%$ of $\mathrm{BD}$ patients suffer from ADHD, suggesting a subgroup of patients where pathologies overlap and presumably risk genes are shared (Nierenberg et al, 2005; Tamam et al, 2008). aADHD patients feature a distinct personality profile, with increased scores in the personality domain Neuroticism as measured with the NEO PI-R (Costa and McCrae, 1992), while Conscientiousness, Extraversion, and Openness are decreased (Jacob et al, 2007). Similarly, personality assessment using the TPQ (Cloninger et al, 1993) reveals that Novelty Seeking and Harm Avoidance are increased in aADHD patients (Jacob et al, 2007). Taken together, the clinical appearance of aADHD represents a complex clinical phenotype. ADHD in children (cADHD) was repeatedly shown to feature a strong genetic component, and a meta-analysis of 20 studies provided a heritability index of 0.76 (Faraone et al, 2005). However, despite this considerable heritability, only a few risk genes with small effects have been identified. This might be due to the facts that cADHD (a) is a heterogeneous clinical condition (with the risk of phenocopies), (b) might feature substantial ethnic background effects, and (c) is a genetically complex disorder with common variants conveying low risk (Franke et al, 2009) as well as individually rare variants with variable penetrance conveying high risk (Becker and Knapp, 2004; Lesch et al, 2011; Williams et al, 2011). aADHD has come into the focus of genetic research more recently, but has also been shown to have a genetic basis (IMpACT Collaboration, submitted). Molecular genetic findings are even more sparse; this might partly be due to the fact that not only risk variants causing disease as such come into play, but also genes modifying the developmental trajectory of the disorder - that is, persistence into adulthood (eg, SLC6A3; (Franke et al, 2010) - may be of importance.

To overcome the problematic issues coming along with candidate gene studies, genome-wide approaches were increasingly used in the last years. These include genomewide linkage analyses, eight of which have been published so far; seven of these were included in a meta-analysis (Zhou et al, 2008). Furthermore, genome-wide association studies (GWAS) were employed to find new risk genes (Franke et al, 2009; Neale et al, 2010a,b). We have previously reported on a pooled GWAS in aADHD (Lesch et al, 2008), where two single-nucleotide polymorphisms (SNPs) in the top 500 localized to the intergenic region between DIRAS2 and SYK on chromosome 9q22.2. The DIRAS2-SYK locus is also supported by two linkage scans, one of which was performed in individuals from the same catchment population as the aADHD GWAS (Romanos et al, 2008), while the other was conducted in the United Kingdom (Asherson et al, 2008). Further interest in this region comes from a pooled GWAS in PD, where $S Y K$ was the number 11 top-locus for cluster B PD (in preparation). Thus, three independent and hypothesis-free studies provide converging evidence that this locus might contribute to the genetic risk for disorders of cognitive control featuring increased impulsivity. DIRAS2 is strongly expressed in the brain, while $S Y K$ is preferentially expressed in peripheral tissues, making DIRAS2 the prime candidate gene at this locus. Intriguingly, in a recent GWAS on cognitive performance of the aging brain, DIRAS2 was associated $\left(p=8 \times 10^{-6}\right)$ with performance in the Boston naming test arguing for a role of DIRAS2 in cognitive processes (Seshadri et al, 2007).

These findings prompted us to conduct a study using large discovery and replication aADHD samples to assess the genetic relevance of DIRAS2 for aADHD. In addition, we attempted generalization of the findings to CADHD and to related disorders of impulsivity. As the discovery samples also were assessed for personality factors, we were also able to test the influence of DIRAS2 on impulsivity-related traits, which might be considered an intermediate phenotype of ADHD.

\section{MATERIALS AND METHODS}

\section{Samples}

Discovery sample: aADHD sample. The sample was previously described in greater detail (Franke et al, 2010; Jacob et al, 2007). Briefly, 600 patients were extensively examined using a semi-structured interview as well as by applying the SCID-I and SCID-II. Personality assessment was done using NEO PI-R and TPQ. Eligibility criteria for the study were aADHD according to the diagnostic criteria of DSM-IV (Diagnostic and Statistical Manual for Mental Disorders), onset before the age of 7 years, life-long persistence, current diagnosis, and age at recruitment between 18 and 65 years. Exclusion criteria were a current diagnosis of drug/alcohol abuse/dependence (before detoxification/withdrawal), or a lifetime diagnosis of $\mathrm{BD}$, schizophrenia, and mental retardation. A more detailed sample description including the used rating scales can be obtained from Supplementary Methods. The control group was recruited from the Lower Franconia area in Germany and consisted of 420 healthy volunteers. This sample of volunteers was also used as a control for the association analysis for PD and BD.

Family-based childhood ADHD sample. Detailed information on the sample was reported previously (Renner et al, 2008). Briefly, total sample size was 166 families, of whom 109 were trios, 42 quartets, and 15 were multi-sibling families. We recruited families with at least one child affected by ADHD, combined type according to DSM-IV as diagnosed by a structured interview with the parents 
(KIDDIE-SADS), parent and teacher questionnaires and direct clinical observation. The index child was aged 8 years or above. Exclusion criteria were IQ below 80, co-morbid autistic or somatic disorders, primary affective disorders including BD, Tourette's syndrome, psychotic, or other severe primary psychiatric disorders.

IMpACT aADHD samples. The aADHD replication samples have been described in detail elsewhere (Franke et al, 2010). The total sample included 1035 aADHD patients and 1381 controls and originated from the Netherlands (285 patients and 508 controls), Norway (497 cases and 562 controls), Spain (253 patients and 311 controls), and was made available within the framework of the IMpACT consortium. All patients were evaluated by experienced psychiatrists and diagnosed with persistent ADHD according to DSM-IV criteria and also using standardized rating scales. Consensus eligibility criteria for this study across all study sites were a diagnosis of ADHD according to the diagnostic criteria of DSM-IV, onset before the age of 7 years by retrospective diagnosis (which was confirmed by a family member, wherever possible), life-long persistence and current diagnosis. Further information on the samples as well as used rating scales can be obtained from Supplementary Methods.

$P D$ sample. This sample has been described in detail previously (Jacob et al, 2005). A total of 622 inpatients and outpatients of the Department of Psychiatry and Psychotherapy, University of Würzburg with PDs according to DSM-IV criteria participated in the study. Patients were drawn from the general population of hospital patients; every patient with a clinical diagnosis of PD treated in the department from 2000 to 2004 was approached and asked to participate in the study. Inclusion criteria were PD according to DSM-IV (antisocial, histrionic, borderline, narcissistic, avoidant, dependent, and obsessive-compulsive PD) and age between 18 and 60 years. Exclusion criteria were medical conditions and lifetime diagnosis of schizophrenia or other psychotic disorders. The SCID-II was used to diagnose PDs, and PDs were allocated to cluster B and C operationalized as follows: cluster B (77\%) (dramaticemotional) encompasses antisocial, borderline, histrionic, and narcissistic PDs. Cluster C (37\%) (anxious-fearful) includes avoidant, dependent, and obsessive-compulsive PDs. Furthermore, co-morbid axis-I disorders were assessed using the SCID-I as well as extensive clinical interview. Thereby, a lifetime diagnosis of BD (type I and II) was established in 17 of the $622 \mathrm{PD}$ patients $(2.7 \%)$. The assessment of the PDs, including all psychometric testing, was performed by a single experienced psychiatrist (CPJ).

$B D$ sample. This study investigated a sample described previously in detail (Scholz et al, 2010). This sample consisted of 170 unrelated bipolar patients (mean age $53 \pm 14$ years, $68 \%$ female), from the German Lower Franconia area for whom an ICD-10 diagnosis was established by means of an extensive, semi-structured interview analogous to the AMDP interview (AMDP, 2000) carried out by two experienced psychiatrists at the University of Würzburg. Furthermore, the OPCRIT system was used in these patients (McGuffin et al, 1991). A further 166 unrelated bipolar patients (mean age $43.0 \pm 11.5$ years,
$49 \%$ female), were ascertained according to ICD-10 diagnostic criteria for research (DCR) by means of a semi -structured interview (SCAN ver. 2.1.) at the Center for Psychiatric Research, Århus University Hospital, giving a total number of 336 patients suffering from BD. Co-morbidity data for $\mathrm{BD}$ with aADHD could not be obtained as these patients were ascertained during acute disease episodes where it is not possible to reliably establish a diagnosis of aADHD. None of the subjects showed significant neurological co-morbidity, epilepsy, mental retardation, or other somatic disorders suggesting organic psychosis. Patients with substance-induced disorders were excluded.

\section{In Silico Replication in the SHIP GWAS}

Data from the Study of Health in Pomerania (SHIP) were used (Grabe et al, 2005). The target population comprises adult German residents (20-79 years) in a northeastern German population of 212157 individuals. The net sample comprised 6267 eligible subjects, of which 1859 consecutively recruited subjects were eligible for the present analyses. The assessment of personality traits was performed with the NEO-FFI-30 (Korner et al, 2008). The sample was genotyped using the Affymetrix Genome-Wide Human SNP Array 6.0. As most of the DIRAS2 SNPs genotyped in the discovery sample (Table 1) were not captured, genotype data were derived from standard imputation procedures using the software IMPUTE v0.5.0 based on HapMap II.

All cases and controls of the above samples were of self-reported German (or Danish, respectively) ancestry. The study was approved by the respective ethics committees of the participating centers and complied with the latest version of the Declaration of Helsinki. Written informed consent was provided by all participants.

\section{Marker Selection and Genotyping}

In all, 14 SNPs were selected, which tagged the promoter region, the $5^{\prime}$ and $3^{\prime}$ UTRs as well as the transcribed exon of the DIRAS2 gene, spanning a chromosomal region of $33 \mathrm{~kb}$. Marker selection was performed using the Tagger algorithm (parameter: pair-wise tagging only, $r^{2}$ threshold $=0.8$, minor allele frequency (MAF) above 5\%) as implemented in HaploView (www.broad.mit.edu/mpg/haploview) (Figure 1; the haplotype structure was comparable in each sample). SNPs were genotyped using the Sequenom MassArray system (Sequenom, San Diego, CA) coupled to a Bruker Autoflex mass spectrometer (Bruker Daltonics, Bremen, Germany) according to the manufacturer's instructions. PCR was performed using iPlex chemistry as recommended in the MassArray iPlex standard operating procedure. Primer sequences can be obtained on request. rs 12350590, located proximal of the DIRAS2 gene, and rs 9695432 in intron 1, were excluded from further analyses because of MAF $<0.01$ in the discovery sample.

\section{Statistical Analysis}

For all samples, we analyzed only SNPs passing quality check which was defined by MAF $>0.01$, call rate $>90 \%$ and 


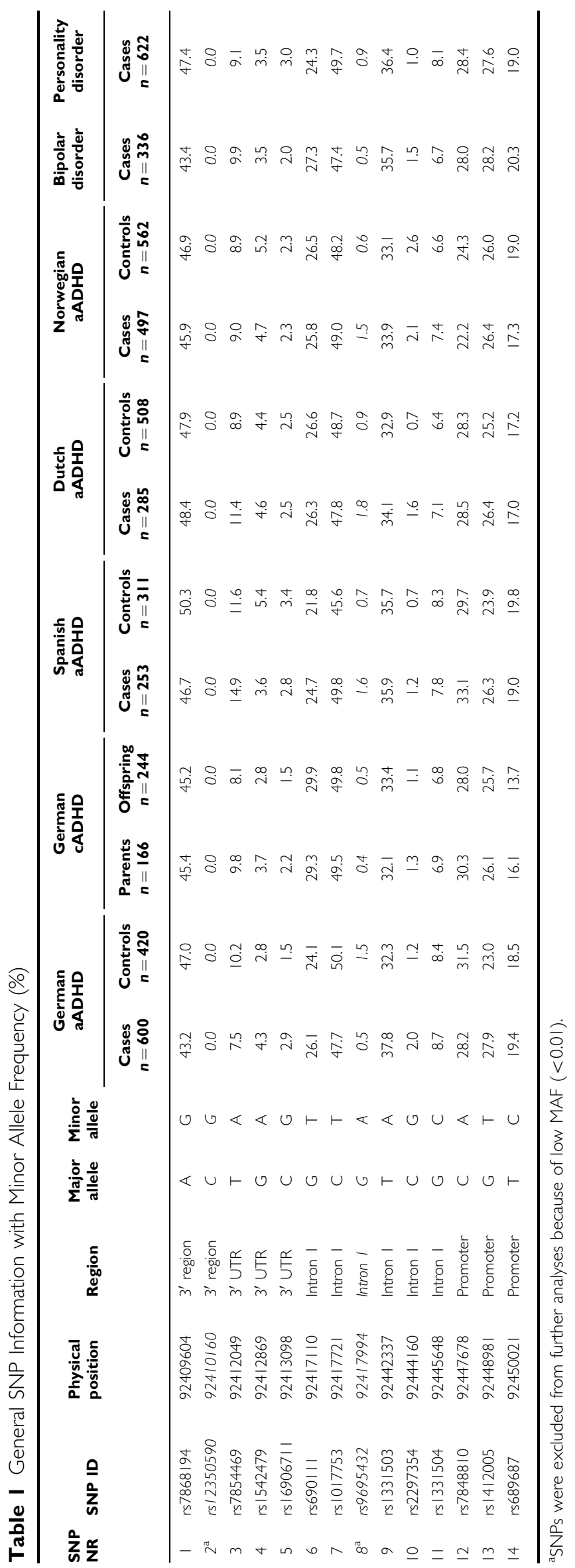

$p$-value of $\chi^{2}$ test for Hardy-Weinberg equilibrium $>0.01$ in controls or parents. Inconsistent genotypes in the family sample were revealed and removed by means of PedCheck (O'Connell and Weeks, 1998). Generalized linear models (GLM) implemented in the R function $\operatorname{glm}()$ were carried out to test for an association between SNP genotype as independent variable and a trait of interest as dependent variable (binary: ADHD, bipolar, cluster $\mathrm{B}$ and $\mathrm{C} \mathrm{PD}$; quantitative: NEO-PI $\mathrm{R}$ (Neuroticism, Extraversion, Openness, Agreeableness, and Conscientiousness) and TPQ (Novelty Seeking, Harm Avoidance, and Reward Dependence)). Binomial logit link or Gaussian identity link was used for binary or quantitative traits, respectively. We also performed Fisher's exact test in order to assure the asymptotic test results of $\operatorname{glm}()$. Family-based association analysis was carried out using pedigree disequilibrium test (sumPDT, Martin et al, 2000) to assess whether an allele is more frequently transmitted to affected offspring by taking relationships between the offspring into account.

We used the omnibus test implemented in famhap (10000 simulations; (Becker and Knapp, 2004) to test the global hypothesis that association with ADHD may be contributed by at least one of the estimated haplotypes. The LD blocks were defined by pairwise $\mathrm{D}^{\prime}>0.65$ assessed using Haploview. After the haplotype ACGCTT from block 2 (consisting of SNPs rs1331503, rs2297354, rs1331504, rs7848810, rs1412005, and rs689687) was identified as the sole haplotype consistently increasing risk across all samples, a haplotype-specific association test using Unphased (Dudbridge, 2008) was carried out.

For the meta-analysis, we assumed a common effect of the rs1412005 T allele and the ACGCTT haplotype on the risk for

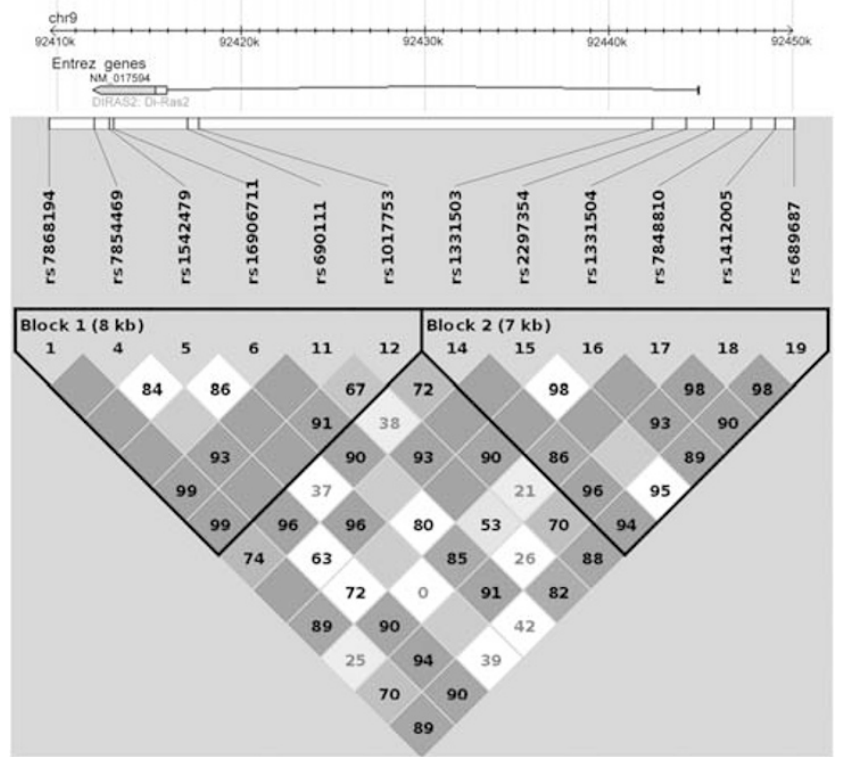

Figure I LD plot of DIRAS2 aligned to the gene structure and the significant SNPs from all samples. Please note that the LD plot is not exactly matched to the SNPs, as precise scaling was not possible: rs9695432 is the last SNP of block I while rs I 33 I 503 is the first SNP of block 2. rs I 69067 I I is associated with $\mathrm{AADHD}$, cluster C PD, cluster B PD, and Harm Avoidance in PD; rs9695432 with aADHD and personality domains; rs I33 I503 with aADHD and personality domains; rs2297354 with aADHD and personality domains; rs7848810 with aADHD, cluster $\mathrm{B} P D, B D$, and personality domains; and rs/4I2005 with aADHD and personality domains. 
ADHD, as there was no effect heterogeneity across all four considered aADHD case-control samples ( $p$-value $=0.43$ and 0.29 , respectively, Woolf's test for heterogeneity, obtained with the $\mathrm{R}$ function meta. $\mathrm{MH}())$. A joint analysis assessing the common OR was done by means of GLM described above, adjusted for fixed country effect. This approach was also applied to the total data set including all patients (aADHD, bipolar, and PDs) and controls in order to estimate the cross disorder effects of DIRAS2 SNPs.

Unless otherwise specified, $p$-values are two-sided $p$-values unadjusted for multiple testing. The significance level was set to 0.05 . Our study primarily aimed at detecting ADHD-associated SNP variants of DIRAS2, whereas analyses for other traits were exploratory. Therefore, we used the Bonferroni method to calculate $p$-values adjusted for testing multiple SNPs and haplotypes for association with ADHD.

Power calculations were performed under a two-sided nominal significance level of 0.05 using QUANTO version 1.0 (http://hydra.usc.edu/gxe). We assumed an effect size between 1.1 and 1.5 for the rs1412005 $\mathrm{T}$ allele and between 1.3 and 3.0 for the ACGCTT haplotype. By given disease prevalence of $5 \%$, we computed the power to detect these variants in the replication sample consisting of 1035 aADHD patients and 1381 controls from IMpACT, under a frequency of $25 \%$ and $5 \%$ for the rs $1412005 \mathrm{~T}$ allele and the ACGCTT haplotype, respectively. All assumptions nearly corresponded with nominal $95 \%$ confidence intervals and frequencies estimated from the discovery sample.

\section{RESULTS}

\section{Association of DIRAS2 with aADHD}

Table 1 displays information about functional and physical location of each SNP in/near DIRAS2. In the discovery sample of 600 aADHD patients and 420 controls from Germany, 4 out of 12 investigated SNPs had $p<0.05$ in the association test with aADHD (Table 2). An empirical test at the gene level for association of at last one of 12 SNPs yielded a $p=0.0127$ (10 000 permutations). Upon haplotype testing, both LD blocks (Figure 1) showed evidence of association with nominally significant $p$-values $(p=0.006$ for block 1 and $p=0.033$ for block 2).

Replication in 1035 aADHD patients and 1381 controls from IMpACT did not show any significant SNP association (all $p>0.07$; Table 2). Also for each individual country, we found neither an association on the single marker $(p>0.1$, Supplementary Table 1) nor on the haploblock level $(p>0.09)$. However, consistency of the effect size estimates was observed indicating increased aADHD risk for carriers of the A allele of rs1331503, the T allele of rs1412005, and the haplotype ACGCTT from block 2 (consisting of SNPs rs1331503, rs2297354, rs1331504, rs7848810, rs1412005, and rs689687; Table 3) across the four samples. Association testing for this specific haplotype provided a significant result in the initial sample from Germany $(p=0.0006)$, which replicated in the additional independent combined samples from IMpACT $(p=0.026)$. To further explore the association of DIRAS2 with aADHD, we performed five meta-analyses, for the best four SNPs of the discovery sample (rs7854469, rs16906711, rs1331503, and rs1412005) and for the risk haplotype ACGCTT, in all 1635 aADHD cases and 1801 controls from Germany, the Netherlands, Spain, and Norway taking population strata into account. A $p=0.04$ was found for the rs $1412005 \mathrm{~T}$ allele in the association test with aADHD ( $p$-value adjusted for five tests: $0.22 ; \mathrm{OR}=1.12,95 \%$ CI: $1.00-1.25$; Figure 2). The risk haplotype ACGCTT $(9.0 \%$ in cases and $6.5 \%$ in controls) was associated with an increased risk for aADHD, and this effect was statistically significant even after adjusting for multiple testing $(p=0.00028, p$-value adjusted for five tests: $0.001, \mathrm{OR}=1.45,95 \%$ CI: $1.19-1.77$; Figure 3). This haplotype contains the $\mathrm{T}$ allele of SNP rs1412005.

\section{DIRAS2 and Childhood ADHD}

In order to extend our findings for DIRAS2 to cADHD, we also analyzed 166 independent nuclear families with offspring affected by cADHD. The rs1412005 $\mathrm{T}$ allele was

Table 2 SNP Results of Case-Control-Based Association Analyses for aADHD

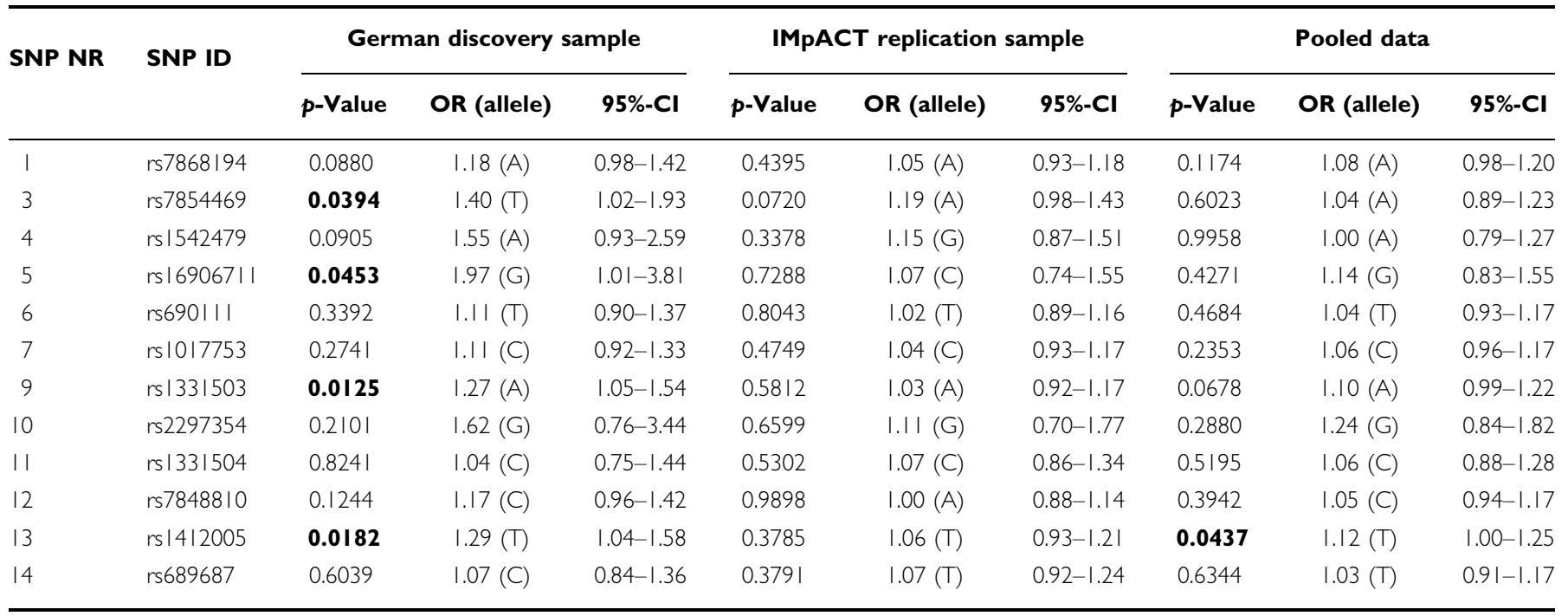

Nominal $p$-values $<0.05$ are in bold print. 
Table 3 Association Analysis of the ACGCTT Haplotype ${ }^{a}$ for the Studied aADHD Case-Control Samples

\begin{tabular}{|c|c|c|c|c|c|}
\hline \multirow{2}{*}{ Sample } & \multicolumn{2}{|c|}{ Frequency: $N$ (\%) } & \multicolumn{3}{|c|}{ Association test } \\
\hline & Cases & Controls & $p$-Value & OR & $95 \%-\mathrm{Cl}$ \\
\hline Germany & 89 (8.94) & $30(4.57)$ & 0.0006 & 2.05 & $1.34-3.14$ \\
\hline The Netherlands & $46(9.54)$ & $66(7.45)$ & 0.1815 & 1.31 & $0.88-1.94$ \\
\hline Spain & $28(6.48)$ & $25(4.46)$ & 0.1815 & 1.48 & $0.85-2.58$ \\
\hline Norway & $76(10.08)$ & $78(8.35)$ & 0.2213 & 1.23 & $0.88-1.71$ \\
\hline
\end{tabular}

a Haplotype consists of SNPs rs I 33 I 503, rs2297354, rs I 33 I 504, rs78488 I0, rs|4|2005, and rs689687.

not significantly over-transmitted $(p=0.65)$, but the $\mathrm{C}$ allele of the neighboring SNP rs7848810-also a promoter SNP - was ( $p=0.046$, transmission rate: $53 \%$; Table 4$)$. Haplotype analysis provided evidence of significant association of the haploblock $2(p=0.0231)$ with a $p$-value for the specific haplotype ACGCTT slightly exceeding 0.05 ( $p=0.0692$, frequency among transmitted $v s$ nontransmitted haplotypes: $11 \%$ vs $6.5 \%, \mathrm{OR}=2.01,95 \% \mathrm{CI}$ : $0.80-5.00)$. rs7848810 was also associated with co-morbid dyslexia $(p=0.015)$.

\section{Expanding the Phenotype: Association of DIRAS2 with PDs and BD}

To examine whether DIRAS2 is specifically linked to ADHD or whether the association generalizes to disorders sharing the feature of impulsivity and emotional instability (independent of co-morbidity with ADHD), we performed association analyses in two further independent samples: patients suffering from PDs and BD. We found nominal evidence of association of rs 1412005 , the SNP implicated in aADHD (see above), with cluster B PD $(p=0.031)$ and BD $(p=0.026)$. The OR of the T allele for both disorders was estimated at around 1.26 (Table 5). The $\mathrm{T}$ allele also tended toward increasing risk for cluster C PD $(p=0.086)$ with the same OR estimate of 1.26 .

\section{DIRAS2 Relates to Personality Factors Linked to aADHD}

The Würzburg aADHD and PD samples had also been tested for personality dimensions, as assessed by two questionnaires (NEO-PI R: Neuroticism, Extraversion, Openness to Experience, Agreeableness, and Conscientiousness; and TPQ: Novelty Seeking, Harm Avoidance, and Reward Dependence). Given the association of DIRAS2 variants with PDs (see above), and considering that aADHD goes along with a distinct personality profile (Jacob et al, 2007), we additionally investigated whether personality scores may also be affected by DIRAS2 variants. Table 6 represents all nominally significant association results. In the aADHD sample, associations with $p<0.05$ were found for Neuroticism, Extraversion, Agreeableness, Conscientious$n e s s$, and Harm Avoidance ( $p$-values between 0.002 and 0.033). The three SNPs rs1331503, rs1412005, and rs689687, which were associated with these personality traits, are located in haplotype block 2 and the transcriptional control region of the gene. In the PD sample, nominal evidence of
The Effects of the rs1412005 T Allele

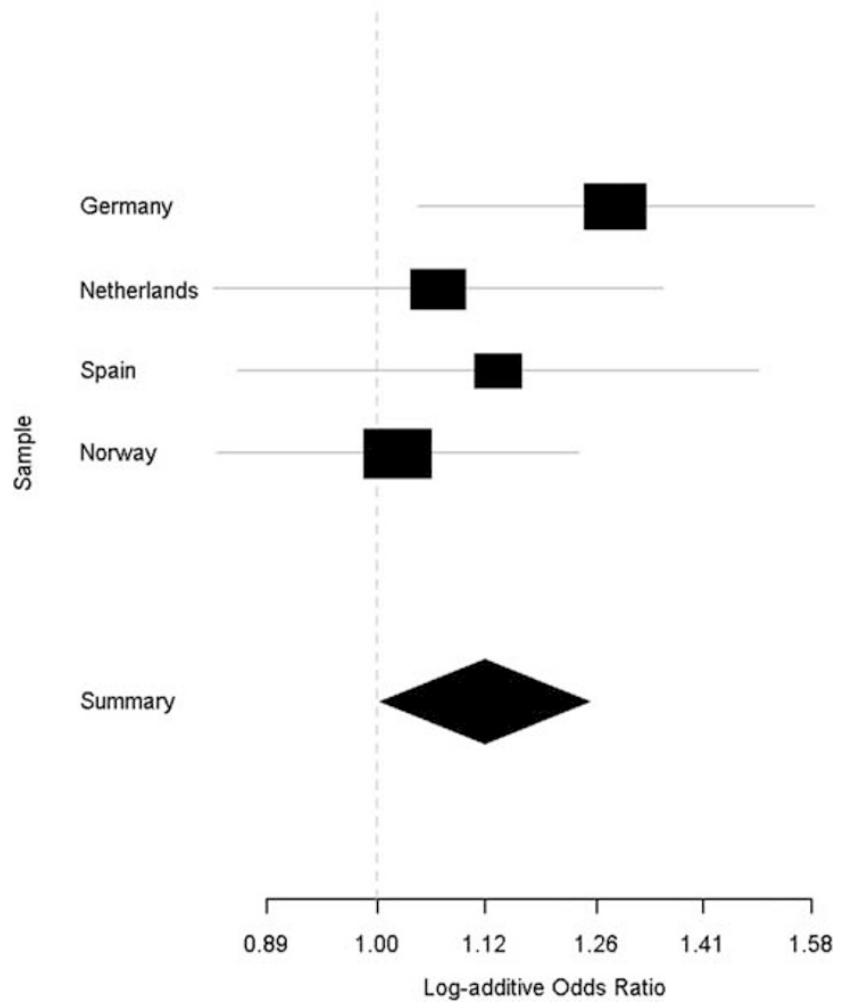

Figure 2 Forest plot of the rs/4/2005 T allele for the aADHD case-control association study.

The Effects of the ACGCTT Haplotype

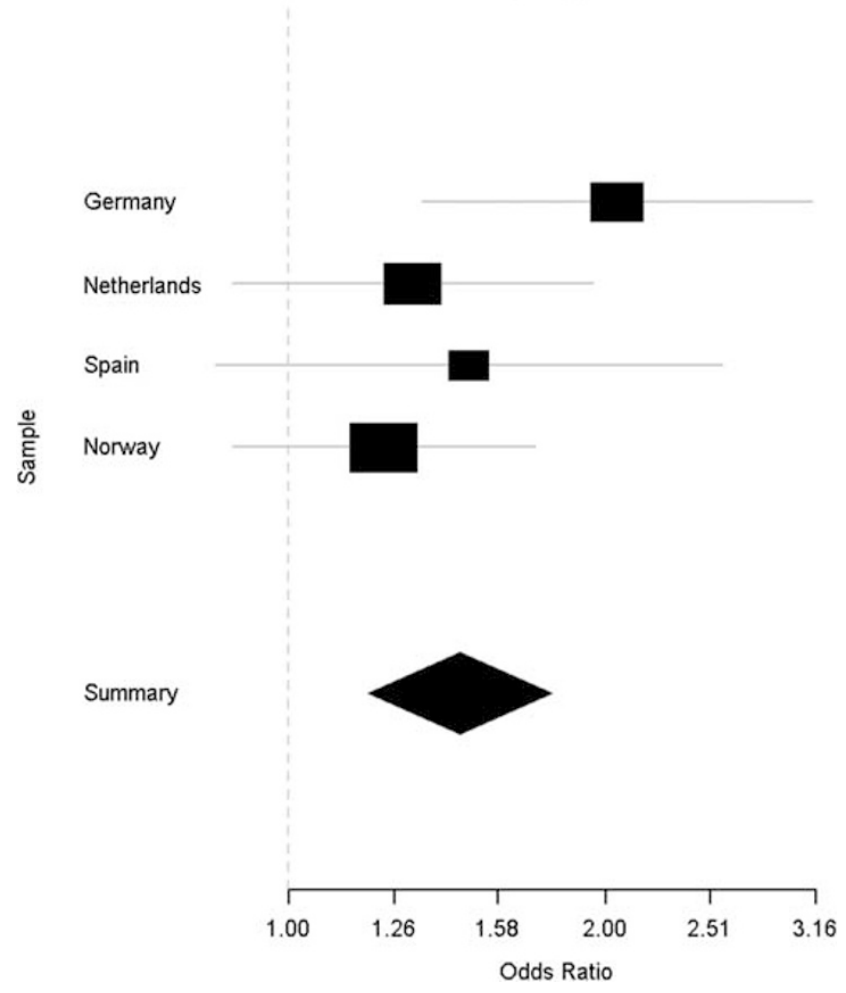

Figure 3 Forest plot of the ACGCTT haplotype for the aADHD case-control association study. 
association was found for Extraversion, Agreeableness, Conscientiousness, Novelty Seeking, and Harm Avoidance ( $p$-values between 0.008 and 0.040 ; Table 6). In comparison with the aADHD sample, different SNPs showed association in this sample and almost all of them are located in the haplotype block 1. However, rs7854469 was associated both with aADHD (see above) and the ADHD-relevant personality trait Extraversion ( $p=0.0075$, Table 6) in PD. Also the aADHD-associated SNP rs7848810 was associated with TPQ Novelty Seeking $(p=0.0396$, Table 6) and NEO PI-R Conscientiousness ( $p=0.0123$, Table 6$)$ in the PD sample.

Owing to the pitfalls of multiple testing, inevitable in personality genetics, replication attempts are paramount. We have thus analyzed 1859 subjects from a general population study (SHIP) who have been examined for personality factors; apart from rs7848810, SNPs had to be

Table 4 Results of Family-Based Association Analysis for cADHD

\begin{tabular}{|c|c|c|c|c|}
\hline \multirow{2}{*}{ SNP NR } & \multirow{2}{*}{ SNP ID } & \multicolumn{2}{|c|}{ Over-transmission } & \multirow{2}{*}{$\begin{array}{c}\text { PDT } \\
\text { p-Value }\end{array}$} \\
\hline & & Allele & Rate (\%) & \\
\hline I & rs7868194 & G & 50.9 & 0.6507 \\
\hline 3 & rs7854469 & $\mathrm{T}$ & 50.8 & 0.2059 \\
\hline 4 & rs 1542479 & G & 50.5 & 0.1779 \\
\hline 5 & rs|69067|I & C & 50.2 & 0.3711 \\
\hline 6 & rs6901।I & G & 50.9 & 0.4982 \\
\hline 7 & rsI017753 & $\mathrm{T}$ & 50.9 & 0.6911 \\
\hline 9 & rs|33|503 & A & 52.2 & 0.4245 \\
\hline 10 & rs2297354 & C & 50.3 & 0.2253 \\
\hline 11 & rs|331504 & G & 50.1 & 0.8997 \\
\hline 12 & rs7848810 & C & 52.8 & 0.0463 \\
\hline 13 & rs|4|2005 & $\mathrm{T}$ & 51.4 & 0.6565 \\
\hline 14 & rs689687 & $T$ & 51.3 & $0.1456^{\mathrm{a}}$ \\
\hline
\end{tabular}

${ }^{\mathrm{a} C a l l}$ rate $=77 \%$.

Nominal $p$-values $<0.05$ is in bold print. imputed from data acquired on the Affymetrix Human SNP Array 6.0 platform. In doing so, rs16906711 was found to be associated with NEO PI-R Neuroticism ( $p=0.046$, Supplementary Table 2), and rs1331504 with NEO PI-R Conscientiousness ( $p=0.002$, Supplementary Table 2$)$.

\section{DISCUSSION}

On the basis of the results of four hypothesis-free approaches converging on the chromosomal locus 9q22.2 - two linkage scans for CADHD, a pooled GWAS of aADHD, and a pooled GWAS of PD - we examined the DIRAS2 gene by genotyping 14 markers, selected to tag the promoter region, the $5^{\prime}$ and $3^{\prime}$ UTRs and the only coding exon of the gene, in a total of seven independent patient samples. ADHD findings replicated across aADHD samples, with meta-analytic treatment implicating the ACGCTT haplotype (which spans the regulatory region of DIRAS2) and one of the SNPs contributing to this haplotype (rs1412005, T allele), and generalized to ADHD in childhood. However, after correction for multiple testing, only the association of the haplotype remained significant arguing that the association of DIRAS2 with ADHD most likely is not due to one of the examined single markers but rather to a variant segregating with this risk haplotype. Furthermore, expanding the phenotype to impulsivity disorders and impulsivity-related traits pointed toward a role for DIRAS2. Although two of the associated SNPs are located in the $3^{\prime}$ UTR and potentially involved in expression regulation (rs7854469 and rs16906711), two others (rs7848810 and rs1412005) were located upstream of the transcription start site (Figure 1). Scanning of the transcriptional control region SNPs for putative overlapping transcription factor-binding motifs revealed that the sequence carrying the major allele of rs7848810 contained predicted response elements for PBX1 and for the PBXHOXA9 dimer. PBX1 is known for its developmental regulation of neuropeptide $\mathrm{Y}$ expression (Mayer et al,

Table 5 Results of Case-Control Association Analysis for Co-Morbid Bipolar Disorder and Personality Disorders

\begin{tabular}{|c|c|c|c|c|c|c|c|c|c|c|}
\hline \multirow{2}{*}{ SNP NR } & \multirow{2}{*}{ SNP ID } & \multicolumn{3}{|c|}{ Biplolar } & \multicolumn{3}{|c|}{ Cluster B } & \multicolumn{3}{|c|}{ Cluster C } \\
\hline & & $p$-Value & OR (allele) & $95 \%-\mathrm{Cl}$ & $p$-Value & OR (allele) & $95 \%-\mathrm{Cl}$ & $p$-Value & OR (allele) & $95 \%-\mathrm{Cl}$ \\
\hline 1 & rs7868|94 & 0.1794 & $1.15(\mathrm{~A})$ & $0.94-1.42$ & 0.9272 & 1.01 (G) & $0.84-1.22$ & 0.3893 & I.I | (G) & $0.88-1.39$ \\
\hline 3 & rs7854469 & 0.8687 & $1.03(\mathrm{~T})$ & $0.73-1.44$ & 0.0868 & $1.35(\mathrm{~T})$ & $0.96-|.9|$ & 0.5588 & $1.12(\mathrm{~A})$ & $0.77-1.63$ \\
\hline 4 & rs I 542479 & 0.4349 & $1.26(\mathrm{~A})$ & $0.70-2.26$ & 0.5663 & $1.17(\mathrm{~A})$ & $0.68-2.00$ & 0.3782 & $1.34(\mathrm{~A})$ & $0.70-2.56$ \\
\hline 5 & rs |69067|| & 0.4935 & $1.32(\mathrm{G})$ & $0.59-2.94$ & $0.0454^{a}$ & $1.95(G)$ & $1.01-3.75$ & $0.0220^{a}$ & $2.33(G)$ & $1.13-4.80$ \\
\hline 6 & rs6901।I & 0.1752 & I. $18(\mathrm{~T})$ & $0.93-1.50$ & 0.7622 & $1.03(\mathrm{~T})$ & $0.83-1.29$ & 0.8047 & $1.03(\mathrm{G})$ & $0.79-1.36$ \\
\hline 7 & rs 1017753 & 0.2906 & $1.12(\mathrm{C})$ & $0.91-1.38$ & 0.9950 & $1.00(\mathrm{C})$ & $0.83-\mid .21$ & 0.3930 & $1.10(\mathrm{~T})$ & $0.88-1.39$ \\
\hline 9 & rs|331503 & 0.1923 & $1.16(\mathrm{~A})$ & $0.93-1.44$ & 0.1416 & $1.16(\mathrm{~A})$ & $0.95-1.42$ & 0.1950 & $1.18(\mathrm{~A})$ & $0.92-1.50$ \\
\hline 10 & rs2297354 & 0.6345 & $1.24(\mathrm{G})$ & $0.51-3.02$ & 0.5963 & $1.28(\mathrm{C})$ & $0.51-3.18$ & 0.8929 & 1.07 (G) & $0.38-2.99$ \\
\hline | | & rs|331504 & 0.2404 & $1.27(\mathrm{G})$ & $0.85-1.89$ & 0.6503 & $1.08(\mathrm{G})$ & $0.77-1.53$ & 0.5419 & $1.14(\mathrm{G})$ & $0.74-1.76$ \\
\hline 12 & rs7848810 & 0.1645 & $1.17(\mathrm{C})$ & $0.94-1.47$ & 0.1065 & $1.18(C)$ & $0.96-1.46$ & 0.7176 & $1.05(\mathrm{C})$ & $0.82-1.34$ \\
\hline 13 & rs|4|2005 & 0.0256 & $1.32(\mathrm{~T})$ & $1.03-1.67$ & 0.0305 & $1.27(\mathrm{~T})$ & $1.02-1.59$ & 0.0855 & $1.26(\mathrm{~T})$ & $0.97-1.64$ \\
\hline 14 & rs689687 & 0.3988 & $1.12(\mathrm{C})$ & $0.86-1.47$ & 0.7428 & $1.04(\mathrm{C})$ & $0.81-1.34$ & $0.8|4|$ & $1.04(\mathrm{C})$ & $0.77-1.40$ \\
\hline
\end{tabular}

${ }^{a} \mathrm{pHWE}=0.001$

Nominal $p$-values $<0.05$ are in bold print. 
Table 6 Nominally Significant Results of Association Analysis for Quantitative Personality Traits

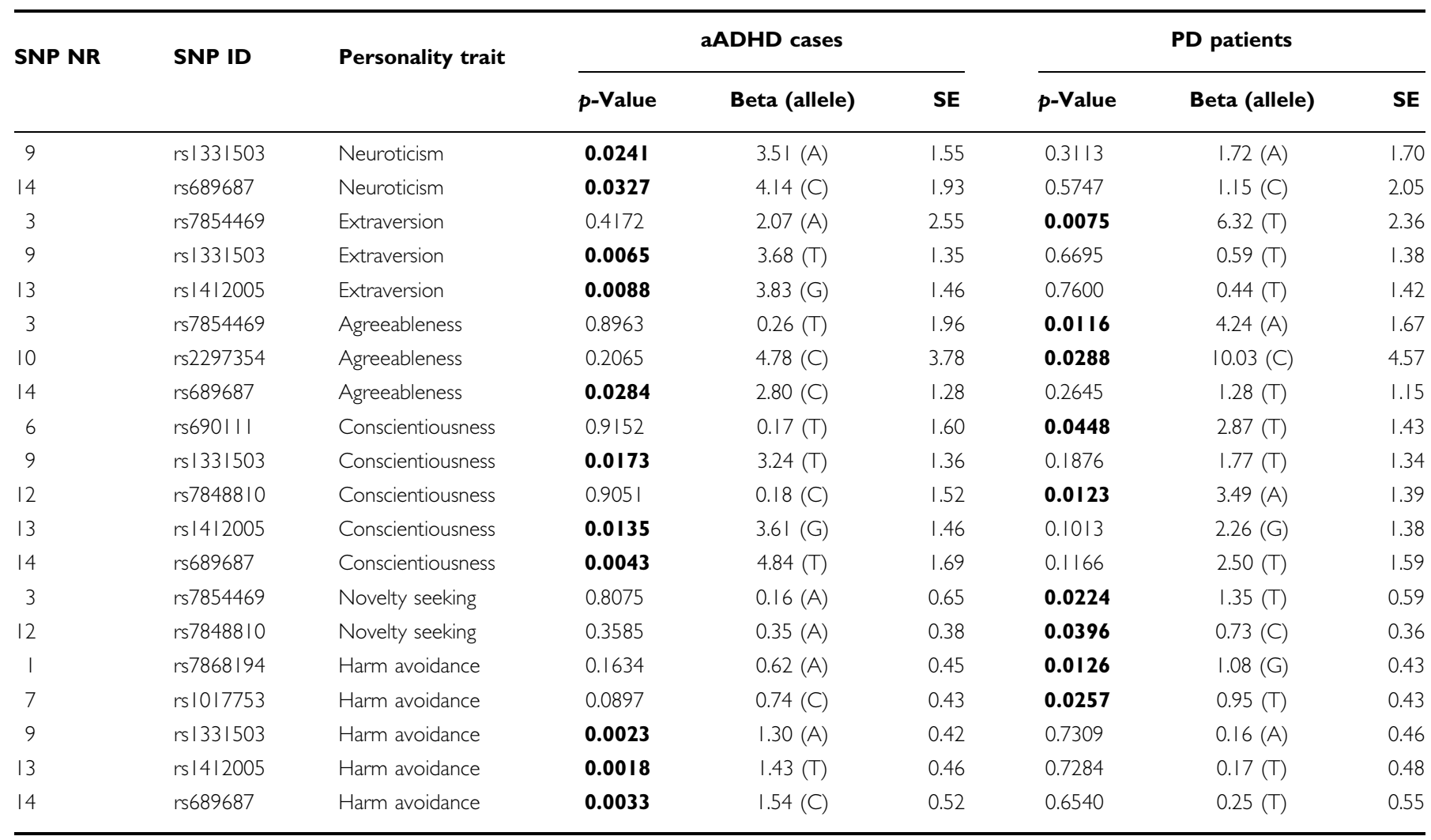

SNPs with $p$-value $<0.05$ in at least one analysis are in bold print.

2003), whereas PBX-HOX dimers generally have important roles in vertebrate development (Moens and Selleri, 2006). Minor allele carriers may therefore have an impaired developmental expression of DIRAS2. Meta-analytic treatment of the aggregated ADHD data provided further evidence especially for ACGCTT haplotype, which includes the rs1412005 $\mathrm{T}$ allele and spans the regulatory region of DIRAS2, to be associated with aADHD. We therefore hypothesize that alterations in the expressional control of DIRAS2 underlie the ADHD association, although further studies have yet to corroborate our findings.

Evidently, association of DIRAS2 is not specific to ADHD, but also occurs for other phenotypes such as bipolar and PDs. A cross disorder analysis for all disorders combined (aADHD, bipolar, and PD) provided an OR effect for the rs1412005 T allele of 1.13 (95\% CI: $1.02-1.26, p=0.02$ ). Given the considerable co-morbidity of these disorders with aADHD, our findings may explain a (although presumably small) part of the genetic variance underlying these clinical observations. Clues to which neurobiological (endo-)phenotype is modulated by DIRAS2 might be obtained from personality genetics, as nine DIRAS2 SNPs were associated with personality domains in aADHD or PD patients. Specifically, aADHD patients carrying the rs1412005 T allele, showing meta-analytic evidence of increasing risk toward aADHD, exhibit on average 3.6 point lower Conscientiousness and 1.4 point higher Harm Avoidance scores than non-carriers (Table 6). Thus, DIRAS2 seems to predispose to a characteristic personality profile going along with ADHD, which might best be characterized by reduced Conscientiousness and increased Harm Avoidance.
However, it has to be noted that associations were quite disparate between samples so that these data can only be considered preliminary ahead of further replication.

What are the molecular mechanisms underlying these association findings? As almost nothing is known about the function of DIRAS2, we can only speculate. The expression profile of DIRAS2 is specific to the brain displaying marked expression in the prefrontal cortex, the anterior cingulate cortex and the amygdala. Although the former two structures are involved in working memory, error detection and executive control - all of which are impaired in ADHD - the latter is also a key component in fear conditioning and anxiety; dampened amygdala response might well lead to increased risk taking behavior. Although DIRAS2s expression pattern endorses a potential pathophysiological role in ADHD and personality, the relevant molecular cascades are elusive. Di-Ras2, the protein encoded by DIRAS2, belongs to the small GTPase Ras family, whose members have roles in cell morphogenesis and neurogenesis. Within the Ras family, Di-Ras2 seems to have unusual biochemical properties (Kontani et al, 2002). Unlike other Ras kinases, it fails to activate the MAP kinase, phosphoinositide 3-kinase or AKT pathways. To date, no effector mechanisms have been described, and due to its specific catalytic characteristics, it is even unclear whether Di-Ras2 is regulated by GTP binding at all. Highly selective expression in raphe serotonin neuron sub-populations (Wylie et al, 2010) points to a role in the regulation of the caudal serotonergic system; however, precise pathways again remain elusive. Despite it being a commonplace statement, in this case further studies are clearly needed to 
even speculate on the neurobiological and behavioral role of Di-ras2.

Several caveats have to be considered in the interpretation of our data. Given the genetic effect sizes (odds ratio) estimated in the discovery sample (1020 German individuals), our replication sample (2416 individuals from IMpACT) had sufficient power to detect the effects of the rs1412005 T allele (59-100\%) and the ACGCTT haplotype $(88-100 \%)$. Despite this fact, there still is a chance of falsepositive findings. This is relevant as most associations on the single marker level would not withstand correction for multiple testing, which becomes an even more prominent issue when examining multi-axial dimensional data such as personality traits. However, as we achieved the essential goal of targeted replication in several independent populations at least for the risk haplotype, and in conjunction with previous evidence from linkage studies and GWAS, we are confident to report a true association. As all nearby ( \pm one megabase) genes are not expressed in the brain, it appears unlikely that genetic variants outside of DIRAS2, but in linkage disequilibrium with the scrutinized polymorphisms are pathophysiologically relevant. We therefore suspect that genetic variation within DIRAS2, in linkage disequilibrium with the interrogated SNPs, are the 'true' underlying risk alleles, a hypothesis that will have to be tested in further studies using re-sequencing approaches followed by functional studies. Finally, as we did not obtain strong positive findings from all samples, small individual sample size, background, epistatic or founder effects or even gene-environment interaction may also have a role explaining that DIRAS2 genetic variants contribute to $\mathrm{ADHD}$ and related phenotypes in some, but not all populations.

In conclusion, converging evidence from several hypothesis-free and hypothesis-driven approaches suggest that DIRAS2 is a candidate gene for ADHD and related behavioral domains. Owing to lack of data on the encoded protein, the underlying molecular mechanisms nevertheless are far from being understood, so that future functional studies will have to bridge the genotype-phenotype gap.

\section{ACKNOWLEDGEMENTS}

This study was supported by the DFG (Grant RE1632/1-1, $/ 1-3$ and $/ 5$ to AR, KFO 125 to AR, CPJ, AW, SW, HS and KPL; SFB 581 to KPL, SFB TRR 58 to AR and KPL; RTG 1252 , to AR and KPL; GR 1912/5-1, to HG), BMBF (IZKF Würzburg, 01KS9603, to KPL; IZKF N-27-N, to AR) and the EC (NEWMOOD LSHM-CT-2003-503474, to KPL). The Norwegian part of the study was supported by the Research Council of Norway and the Western Norway Regional Health Authority (Helse Vest). Financial support for the Spanish part of the study was received from 'Instituto de Salud Carlos III-FIS, Spain' (PI040524, PI041267), 'Fundació La Marató de TV3' (092330/31) and 'Agència de Gestió d'Ajuts Universitaris i de Recerca-AGAUR'(2009SGR00971). MR is a recipient of a 'Ramón y Cajal' contract from 'Ministerio de Ciencia e Innovación' (Spain). The Dutch part of the project was supported by the Hersenstichting Nederland (Fonds Psychische Gezondheid). SHIP is part of the Community Medicine Research net of the
University of Greifswald, Germany, which is funded by the Federal Ministry of Education and Research (Grants no. 01ZZ9603, 01ZZ0103, and 01ZZ0403), the Ministry of Cultural Affairs and the Social Ministry of the Federal State of Mecklenburg-West Pomerania. Genome-wide data have been supported by the Federal Ministry of Education and Research (grant no. 03ZIK012) and a joint grant from Siemens Healthcare, Erlangen, Germany and the Federal State of Mecklenburg-West Pomerania. The University of Greifswald is a member of the 'Center of Knowledge Interchange' program of the Siemens AG. We thank J Romanos, A Boreatti-Hümmer and $M$ Heine for their dedicated assistance in patient recruitment. $\mathrm{T}$ Töpner, $\mathrm{N}$ Steigerwald, G Ortega, J Auer, C Gagel, and N Döring are credited for excellent technical assistance. We thank Mariana Nogueira and Montse Corrales for their involvement in the clinical assessment in Spain, M Dolors Castellar and others from the 'Banc de Sang i Teixits' (Hospital Vall d'Hebron, Barcelona) for their collaboration in the recruitment of control subjects, and Cristina Sánchez-Mora for her participation in the preparation of DNA samples from patients and controls. We thank Marije Boonstra and Marten Onnink for help with patient inclusion, as well as Marlies Naber and Angelien Heister for DNA handling and genotyping. The Dutch controls were derived from the Nijmegen Biomedical Study. Principal investigators of the Nijmegen Biomedical Study are LALM Kiemeney, $M$ den Heijer, ALM Verbeek, DW Swinkels, and B Franke. Finally, we thank all patients, families, and controls for their participation in this study.

\section{DISCLOSURE}

In the past 3 years, Jan K Buitelaar has been a consultant to/ member of advisory board of and/or speaker for Janssen Cilag BV, Eli Lilly, Bristol-Myer Squibb, Organon/Shering Plough, UCB, Shire, Medice and Servier. Sandra Kooij has received research grants from Janssen BV and Shire, and is on the speaker's bureau of Janssen and Eli Lilly. Hans Joergen Grabe has received speakers honoraria from Bristol-Myers Squibb, Eli Lilly, Novartis, Eisai, Wyeth, Pfizer, Boehringer Ingelheim, Servier and travel funds from Janssen-Cilag, Eli Lilly, Novartis, AstraZeneca and the SALUS-Institute for Trend-Research and Therapy Evaluation in Mental Health. Henry Völzke has received research grants by Sanofi-Aventis and the Siemens AG. Susanne Walitza has received lecture honoraria from Janssen Cilag, Lilly and AstraZeneca. Christine Freitag was on the speakers' bureau for Novartis and Janssen-Cilag during the last 3 years. Helmut Schäfer has received a honorarium for a lecture from the European School of Oncology and has been a member of a Data Safety Monitoring Board of Daiichi Sankyo. Andreas Reif has received a research grant from Astra Zeneca. The remaining authors declare no conflict of interest.

\section{REFERENCES}

Arbeitsgemeinschaft, für Methodik und Dokumentation in der Psychiatrie (2000). Das AMDP-System. Manual zur Dokumentation Psychiatrischer Befunde. Göttingen, Germany: Hogrefe. 
Asherson P, Zhou K, Anney RJ, Franke B, Buitelaar J, Ebstein R et al (2008). A high-density SNP linkage scan with 142 combined subtype ADHD sib pairs identifies linkage regions on chromosomes 9 and 16. Mol Psychiatry 13: 514-521.

Becker T, Knapp M (2004). Maximum-likelihood estimation of haplotype frequencies in nuclear families. Genet Epidemiol 27: 21-32.

Cloninger CR, Svrakic DM, Przybeck TR (1993). A psychobiological model of temperament and character. Arch Gen Psychiatry 50: 975-990.

Costa PT, McCrae RR (1992). Revised NEO Personality Inventory (NEO PI-R) and NEO Five Factor Inventory (NEO-FFI) Manual. Psychological Assessment Resources: Odessa.

Dudbridge F (2008). Likelihood-based association analysis for nuclear families and unrelated subjects with missing genotype data. Hum Hered 66: 87-98.

Faraone SV, Biederman J, Spencer T, Wilens T, Seidman LJ, Mick E et al (2000). Attention-deficit/hyperactivity disorder in adults: an overview. Biol Psychiatry 48: 9-20.

Faraone SV, Perlis RH, Doyle AE, Smoller JW, Goralnick JJ, Holmgren MA et al (2005). Molecular genetics of attentiondeficit/hyperactivity disorder. Biol Psychiatry 57: 1313-1323.

Franke B, Neale BM, Faraone SV (2009). Genome-wide association studies in ADHD. Hum Genet 126: 13-50.

Franke B, Vasquez AA, Johansson S, Hoogman M, Romanos J, Boreatti-Hummer A et al (2010). Multicenter analysis of the SLC6A3/DAT1 VNTR haplotype in persistent ADHD suggests differential involvement of the gene in childhood and persistent ADHD. Neuropsychopharmacology 35: 656-664.

Grabe HJ, Volzke H, Ludemann J, Wolff B, Schwahn C, John U et al (2005). Mental and physical complaints in thyroid disorders in the general population. Acta Psychiatr Scand 112: 286-293.

Halmoy A, Halleland H, Dramsdahl M, Bergsholm P, Fasmer OB, Haavik J (2010). Bipolar symptoms in adult attention-deficit/ hyperactivity disorder: a cross-sectional study of 510 clinically diagnosed patients and 417 population-based controls. J Clin Psychiatry 71: 48-57.

Jacob CP, Muller J, Schmidt M, Hohenberger K, Gutknecht L, Reif A et al (2005). Cluster B personality disorders are associated with allelic variation of monoamine oxidase A activity. Neuropsychopharmacology 30: 1711-1718.

Jacob CP, Romanos J, Dempfle A, Heine M, WindemuthKieselbach C, Kruse A et al (2007). Co-morbidity of adult attention-deficit/hyperactivity disorder with focus on personality traits and related disorders in a tertiary referral center. Eur Arch Psychiatry Clin Neurosci 257: 309-317.

Klassen LJ, Katzman MA, Chokka P (2010). Adult ADHD and its comorbidities, with a focus on bipolar disorder. J Affect Disord 124: 1-8.

Kontani K, Tada M, Ogawa T, Okai T, Saito K, Araki Y et al (2002). Di-Ras, a distinct subgroup of ras family GTPases with unique biochemical properties. J Biol Chem 277: 41070-41078.

Korner A, Geyer M, Roth M, Drapeau M, Schmutzer G, Albani C et al (2008). [Personality assessment with the NEO-five-factor inventory: the 30-item-short-version (NEO-FFI-30)]. Psychother Psychosom Med Psychol 58: 238-245.

Lesch KP, Selch S, Renner TJ, Jacob C, Nguyen TT, Hahn T et al (2011). Genome-wide copy number variation analysis in attentiondeficit/hyperactivity disorder: association with neuropeptide Y gene dosage in an extended pedigree. Mol Psychiatry 16: 491-503.

Lesch KP, Timmesfeld N, Renner TJ, Halperin R, Roser C, Nguyen TT et al (2008). Molecular genetics of adult ADHD: converging evidence from genome-wide association and extended pedigree linkage studies. J Neural Transm 115: 1573-1585.
Martin ER, Monks SA, Warren LL, Kaplan NL (2000). A test for linkage and association in general pedigrees: the pedigree disequilibrium test. Am J Hum Genet 67: 146-154.

Mayer CM, Cai F, Cui H, Gillespie JM, MacMillan M, Belsham DD (2003). Analysis of a repressor region in the human neuropeptide $\mathrm{Y}$ gene that binds Oct-1 and $\mathrm{Pbx}-1$ in GT1-7 neurons. Biochem Biophys Res Commun 307: 847-854.

McGuffin P, Farmer A, Harvey I (1991). A polydiagnostic application of operational criteria in studies of psychotic illness: development and reliability of the OPCRIT system. Arch Gen Psychiatry 48: 764-770.

Moens CB, Selleri L (2006). Hox cofactors in vertebrate development. Dev Biol 291: 193-206.

Neale BM, Medland S, Ripke S, Anney RJ, Asherson P, Buitelaar J et al (2010a). Case-control genome-wide association study of attention-deficit/hyperactivity disorder. J Am Acad Child Adolesc Psychiatry 49: 906-920.

Neale BM, Medland SE, Ripke S, Asherson P, Franke B, Lesch KP et al (2010b). Meta-analysis of genome-wide association studies of attention-deficit/hyperactivity disorder. J Am Acad Child Adolesc Psychiatry 49: 884-897.

Nierenberg AA, Miyahara S, Spencer T, Wisniewski SR, Otto MW, Simon $\mathrm{N}$ et al (2005). Clinical and diagnostic implications of lifetime attention-deficit/hyperactivity disorder comorbidity in adults with bipolar disorder: data from the first 1000 STEP-BD participants. Biol Psychiatry 57: 1467-1473.

O'Connell JR, Weeks DE (1998). PedCheck: a program for identification of genotype incompatibilities in linkage analysis. Am J Hum Genet 63: 259-266.

Renner TJ, Walitza S, Dempfle A, Eckert L, Romanos M, Gerlach M et al (2008). Allelic variants of SNAP25 in a family-based sample of ADHD. J Neural Transm 115: 317-321.

Romanos M, Freitag C, Jacob C, Craig DW, Dempfle A, Nguyen TT et al (2008). Genome-wide linkage analysis of ADHD using high-density SNP arrays: novel loci at 5q13.1 and $14 \mathrm{q} 12$. Mol Psychiatry 13: 522-530.

Scholz CJ, Jacob CP, Buttenschon HN, Kittel-Schneider S, BoreattiHummer A, Zimmer $M$ et al (2010). Functional variants of TSPAN8 are associated with bipolar disorder and schizophrenia. Am J Med Genet B Neuropsychiatr Genet 153B: 967-972.

Seshadri S, DeStefano AL, Au R, Massaro JM, Beiser AS, Kelly-Hayes $\mathrm{M}$ et al (2007). Genetic correlates of brain aging on MRI and cognitive test measures: a genome-wide association and linkage analysis in the Framingham Study. BMC Med Genet 8(Suppl 1): S15.

Tamam L, Karakus G, Ozpoyraz N (2008). Comorbidity of adult attention-deficit hyperactivity disorder and bipolar disorder: prevalence and clinical correlates. Eur Arch Psychiatry Clin Neurosci 258: 385-393.

Williams NM, Zaharieva I, Martin A, Langley K, Mantripragada K, Fossdal $\mathrm{R}$ et al (2011). Rare chromosomal deletions and duplications in attention-deficit hyperactivity disorder: a genome-wide analysis. Lancet 376: 1401-1408.

Wingo AP, Ghaemi SN (2007). A systematic review of rates and diagnostic validity of comorbid adult attention-deficit/hyperactivity disorder and bipolar disorder. J Clin Psychiatry 68: 1776-1784.

Wylie CJ, Hendricks TJ, Zhang B, Wang L, Lu P, Leahy $\mathrm{P}$ et al (2010). Distinct transcriptomes define rostral and caudal serotonin neurons. J Neurosci 30: 670-684.

Zhou K, Dempfle A, Arcos-Burgos M, Bakker SC, Banaschewski T, Biederman J et al (2008). Meta-analysis of genome-wide linkage scans of attention deficit hyperactivity disorder. Am J Med Genet B Neuropsychiatr Genet 147B: 1392-1398. 